\title{
Breeding Waterfowl in Captiviiły
}

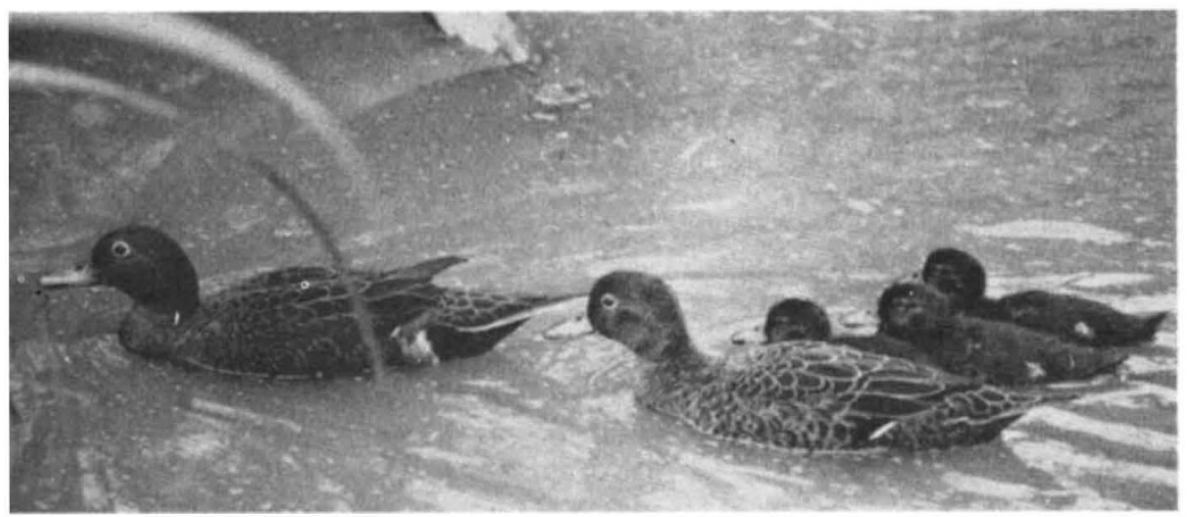

THE indispensable International Zoo Yearbook, (vol. 13, Zoological Society of London, $1973, £ 8$ ) this year has a special section on waterfowl in captivity. The young of the rare New Zealand brown teal (Anas aucklandica chlorotis) shown here were hatched for the first time in captivity at the Wildfowl Trust, Slimbridge. Both parents tend the young, an unusual feature in dabbling ducks.

and Price point out that the microstructure of a block copolymer has four levels of organization: primary chain structure; geometrical conformation of individual chains ; microphase separation and disposition; and "grain structure". This last refers to the existence of regions in which the hexagonal array of polybutadiene microspheres lies on one "lattice", abutting on other regions or "grains" in which the lattice has a different orientation. Lewis and Price have even studied the arrangement of microspheres in the "grain boundaries", which are disturbed just like real grain boundaries in metals. It will certainly benefit the development of materials science that Lewis, a chemist, is now pursuing these studies in a metallurgy department.

Returning to Szwarc's review, a number of illustrations may be found of the advantages of a precise control of the chain structure of a block copolymer. (Note that the living polymer technique allows a polymer of a given gross composition to be produced in a variety of microstructures, corresponding to different block lengths, just as an alloy of fixed composition can be given different microstructures by varied heat-treatments.) The essential physical and chemical properties of the constituent blocks are preserved and not averaged out as in a random copolymer. Thus weak but permeable desalination membranes of cellulose acetate can be stiffened by making a block copolymer with polystyrene, which is more resistant to creep though not by itself permeable.
Again, microstructures with dispersed arrays of rods can be stabilized by dissolving a block copolymer made by Szwarc's method in a suitable, chemically distinct monomer and then rapidly polymerizing the monomer. The rod structure, already existing in the solution, is thus fixed in a matrix which now incorporates the second polymer, and the whole is a form of ultrafine fibre-reinforced polymer, which can be aligned by extrusion.

Szwarc suggests a number of other technologically desirable properties which could be achieved by special forms of highly regular block copolymers, and calls for intense studies in this field. The call is fully justified.

$$
\begin{array}{r}
\text { From our Materials Science } \\
\text { Correspondent }
\end{array}
$$

\section{PROTEINS}

\section{Built to Last}

from our Molecular Biology Correspondent GIVEN the facts of hydrophobia in proteins, one would suppose that the lower limit of chain length to allow the formation of a stable globular conformation would be governed by the number of non-polar side chains needed to generate an "inside" from which water is excluded. So far the smallest proteins that can be categorically said to possess a unique, stable globular structure have fifty to sixty residues, and include the insulins ferredoxins, a family of venom neurotoxins and pancreatic trypsin inhibitor. This last is a remarkable specimen, for in spite of its small size, it exhibits a quite remarkable stability towards denaturants, such as guanidine hydrochloride, renatures from the reduced state with unusual speed and efficiency, and can be kept in solution without loss of activity literally for years. Its sequence and structure are known; it is without histidine or tryptophan, but has three disulphide bonds, providing a rather high cross-link density by the standards of larger proteins. Some remarkable aspects of its stability are now revealed in proton magnetic resonance studies from two laboratories.

Masson and Wüthrich (FEBS Lett., 31, 114 ; 1973) have concentrated their attention on the downfield region of the spectrum, where, below the phenylalanine and tyrosine ring resonances, an extensive series of peaks, mostly corresponding to single protons, and many of them resolved into doublets, are apparent in freshly prepared $\mathrm{D}_{2} \mathrm{O}$ solutions. These are evidently refractory exchangeable hydrogens, presumably of internal peptide groups, showing coupling of the amide proton pairs. Guanidine hydrochloride makes no impression on these resonances until the solution is heated, when, at a sufficiently high temperature, they vanish. Renaturation by diluting out a solution of the protein in dimethyl sulphoxide with $\mathrm{D}_{2} \mathrm{O}$ regenerates the NMR spectrum characteristic of the native state, except for the downfield peaks. Now resonances from shielded exchangeable protons have been observed in fresh solutions of a few other proteins, but they disappear quickly. In pancreatic trypsin inhibitor, on the other hand, of the fifteen observed features of this nature, four diminish noticeably in intensity on a time scale of hours, another six after several days, but the remainder appear to be indefinitely stable. With sufficient effort and ingenuity it may be possible to make specific assignments in terms of Huber's high-resolution X-ray structure. Slow exchange prevails also at $p \mathbf{H} 0.7$, and even in alkali at $p \mathrm{H} 11$ and higher, three of the shielded protons persist. Exchange in the native protein can be provoked by heating, and occurs in minutes at $65^{\circ} \mathrm{C}$, whereas no unfolding can be detected by any of the usual methods until the temperature reaches $85^{\circ} \mathrm{C}$.

Much the same results have been obtained by Karplus, Snyder and Sykes (Biochemistry, 12, 1323; 1973), who waited for four months for the downfield resonances to go away, and at the end of this time the greater number were still there. The usual view of protein conformation is that it allows for a certain measure of undulation or "breathing". By the standards of other globular proteins that of pancreatic trypsin inhibitor is evidently quite remarkably resistant to any such fluctuations, except at high temperature; that is to say, there is an energetic barrier to any kind of unfolding of quite 